\title{
Magnetostriction and electrical resistivity of Mn doped $\mathrm{Fe}_{81} \mathrm{Ga}_{19}$ alloys
}

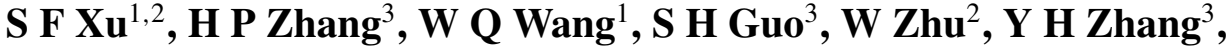 \\ X L Wang ${ }^{3}$, D L Zhao ${ }^{3}$, J L Chen ${ }^{2}$ and G H Wu ${ }^{2,4}$ \\ ${ }^{1}$ Department of Physics, Jilin University, Changchun 130023, People's Republic of China \\ ${ }^{2}$ Beijing National Laboratory for Condensed Matter Physics, Institute of Physics, Chinese Academy of \\ Sciences, Beijing 100080, People's Republic of China \\ ${ }^{3}$ Functional Materials Research Institute, Central Iron and Steel Research Institute, Beijing 100081, \\ People's Republic of China \\ E-mail: userm201@aphy.iphy.ac.cn
}

Received 8 September 2007, in final form 7 November 2007

Published 12 December 2007

Online at stacks.iop.org/JPhysD/41/015002

\begin{abstract}
The paper reports on the effect of substituting $\mathrm{Mn}$ for $\mathrm{Fe}$ on the magnetostrictive and related properties of $\mathrm{Fe}_{81-x} \mathrm{Mn}_{x} \mathrm{Ga}_{19}$ alloys. Samples of composition were prepared in polycrystalline form and were all single phase BCC. It is shown that the magnetostriction observed in

$\mathrm{Fe}_{81} \mathrm{Ga}_{19}$ can be enhanced up to about $11 \%$ by $\mathrm{Mn}$ substitution with a maximum effect at $x=8$. The change in the magnetostrictive coefficient of $\mathrm{Fe}_{81} \mathrm{Ga}_{19}$ with $\mathrm{Mn}$ substitution is accompanied by a monotonic increase in resistivity, being increased by almost a factor of 2 for $x=8$. We also found that the lattice parameter of the alloys increased with the increase in Mn concentration and the Curie temperature decreased monotonically at the same substitution.
\end{abstract}

\section{Introduction}

Magnetostriction is the effect observed when the dimensions of a specimen change with the application of a magnetic field, which has been utilized for many applications such as ultrasonic generation and detection, vibration control and magnetostrictive filters [1,2]. Fe-Ga alloys (Galfenol) have larger magnetostriction coefficient, $\lambda$, than that of $\mathrm{Ni}$ and $\mathrm{Fe}$ and have the advantages of a low temperature coefficient for $\lambda$, low cost compared with rare earth intermetallic compounds, and have the advantage of being operational at low magnetic fields because of their negligible magnetic hysteresis [3-6]. Moreover, unlike many current active materials, such as Terfenol-D and cobalt ferrite, which are brittle and unable to withstand any substantial amount of tension, Galfenol has an appreciable tensile strength and good ductility. Besides being used as actuator materials, Galfenol can also serve as energy harvesting materials for converting mechanical vibrations into voltage signals or electric power. However, the $\mathrm{Fe}-\mathrm{Ga}$ alloys show a relatively low electrical resistivity and eddy current occurs even at modest frequency, which limits the application range.

\footnotetext{
4 Author to whom any correspondence should be addressed.
}

Previously, many efforts were made to increase the electrical resistivity in the Terfenol-D magnetostrictive alloys [7-9]. Co, Ni or Al elements were doped, but high content of the substituting elements is required (such as about 50\% increase with the addition of $10 \% \mathrm{Al}$ ), which leads to a poor magnetostriction. But to our knowledge, works on increasing the electrical resistivity of Galfenol have rarely been reported. Study on intrinsically increasing the electrical resistivity without magnetostriction greatly deteriorated in $\mathrm{Fe}-\mathrm{Ga}$ alloys currently keeps open and attractive.

Mn has much higher resistivity than that of other magnetic metals. Therefore, it is possible to achieve higher resistivity by doping $\mathrm{Mn}$ in $\mathrm{Fe}-\mathrm{Ga}$ alloys [10]. In this work, we present the results of the effects of Mn doping on the structure, electrical resistivity $(\rho)$, the magnetostriction and Curie temperature $\left(T_{\mathrm{C}}\right)$ of $\mathrm{Fe}-\mathrm{Ga}$ alloys. Both high electrical resistivity and even enhanced magnetostrictive properties were obtained in the FeMnGa alloys.

\section{Experimental}

The ingots of $\mathrm{Fe}_{81-x} \mathrm{Mn}_{x} \mathrm{Ga}_{19}(x=0-16)$ were prepared by arc melting the constituent elements, $\mathrm{Fe}$ and $\mathrm{Ga}$ of purity $99.99 \%$ and Mn of purity $99.95 \%$, in an arc furnace under an 


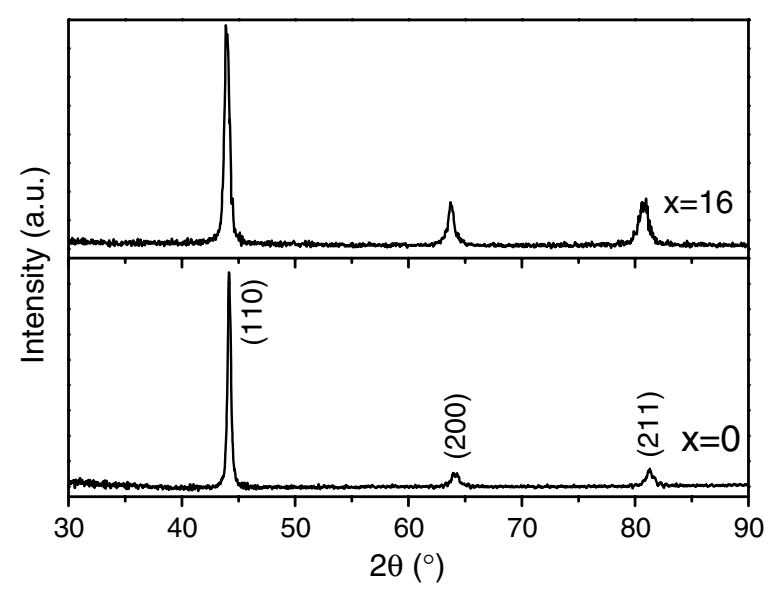

Figure 1. X-ray diffraction patterns of $\mathrm{Fe}_{81-x} \mathrm{Mn}_{x} \mathrm{Ga}_{19}(x=0-16)$.

argon atmosphere. Since Mn has very high vapour pressure leading to the loss of the element, usually $3 \%$ of Mn was added in excess to the total mixture to compensate for the loss [11]. The melting was carried out four times for good homogeneity and the weight loss after the final melting was less than $0.5 \%$. X-ray diffraction measurements were performed by using $\mathrm{Cu} K \alpha$ radiation in a Rigaku D/MAX2200PC diffractometer. The lattice parameters were evaluated by least-squares refinement from powder $\mathrm{x}$-ray diffractograms. The magnetic transition temperatures were measured by ac magnetic susceptibility measurements. The magnetostriction was measured by standard strain gauge on the samples with dimensions of $10 \times 10 \times 2 \mathrm{~mm}^{3}$. In this open magnetic circuit measurement, the demagnetizing effect has not been deducted. Strip specimens for electrical resistivity measurements were cut by using a spark cutting with dimensions of $1 \times 1 \times 15 \mathrm{~mm}^{3}$. The electrical resistivity was measured by using the traditional four-probe technique in the temperature range from 100 to $300 \mathrm{~K}$. In order to reduce the possible measuring errors all specimen surfaces were polished and the dimensions were precisely controlled.

\section{Results and discussion}

Powder X-ray diffraction patterns of $\mathrm{Mn}$ doped $\mathrm{Fe}_{81-x} \mathrm{Mn}_{x} \mathrm{Ga}_{19}$ $(x=0-16)$ arc-melted samples are presented in figure 1 , exhibiting mainly a disordered BCC $A 2$ structure and Mn addition does not change the BCC structure in FeMnGa. According to the phase diagram [12], $\mathrm{Fe}_{81} \mathrm{Ga}_{19}$ alloy is in the disordered BCC $A 2$ phase region at temperatures above $570{ }^{\circ} \mathrm{C}$. On slow cooling to room temperature, ordered $\mathrm{DO}_{3}$ phase was also reported for $\mathrm{Fe}_{81} \mathrm{Ga}_{19}$ alloy $[13,14]$. However, no superlattice reflection peaks of $\mathrm{DO}_{3}$ were detected and $A 2$ structure is believed for the present arc-melted FeMnGa samples. The substitution of $\mathrm{Fe}$ by $\mathrm{Mn}$ atoms changed the peak positions to smaller values of $2 \theta$ by comparing with the corresponding BCC $A 2$ structure of $\mathrm{Fe}_{81} \mathrm{Ga}_{19}$ alloy. So the lattice parameters are seen to slightly increase with increasing Mn concentration in accordance with the larger ionic radius of $\mathrm{Mn}$ compared with $\mathrm{Fe}$ as shown in figure 2(a).

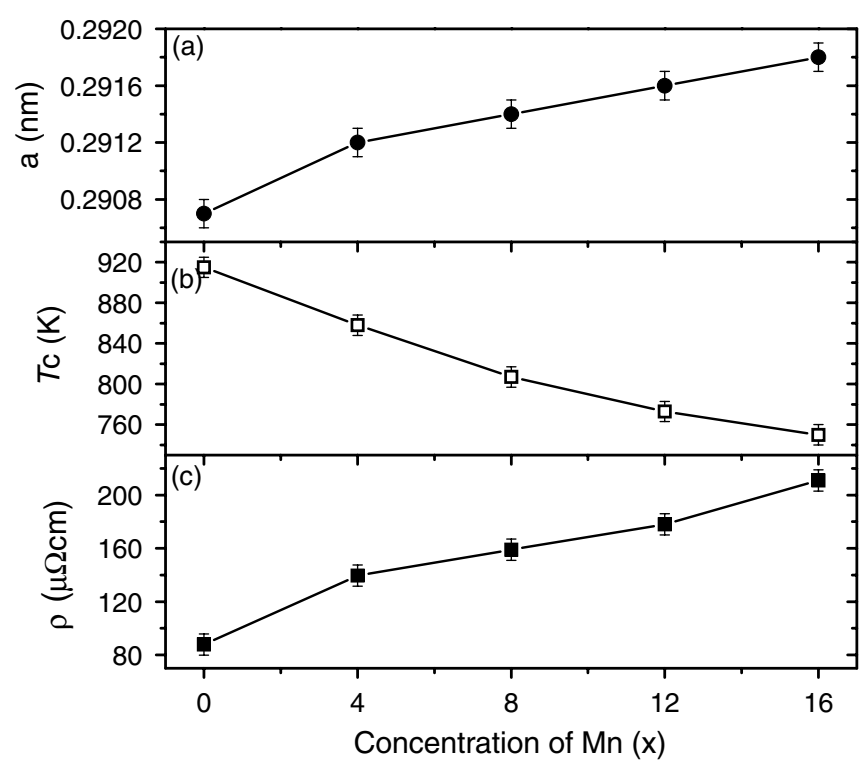

Figure 2. Variation of lattice parameter $(a)$, Curie temperature $(b)$ and resistivity at room temperature $(c)$ for $\mathrm{Fe}_{81-x} \mathrm{Mn}_{x} \mathrm{Ga}_{19}$ with $x$.

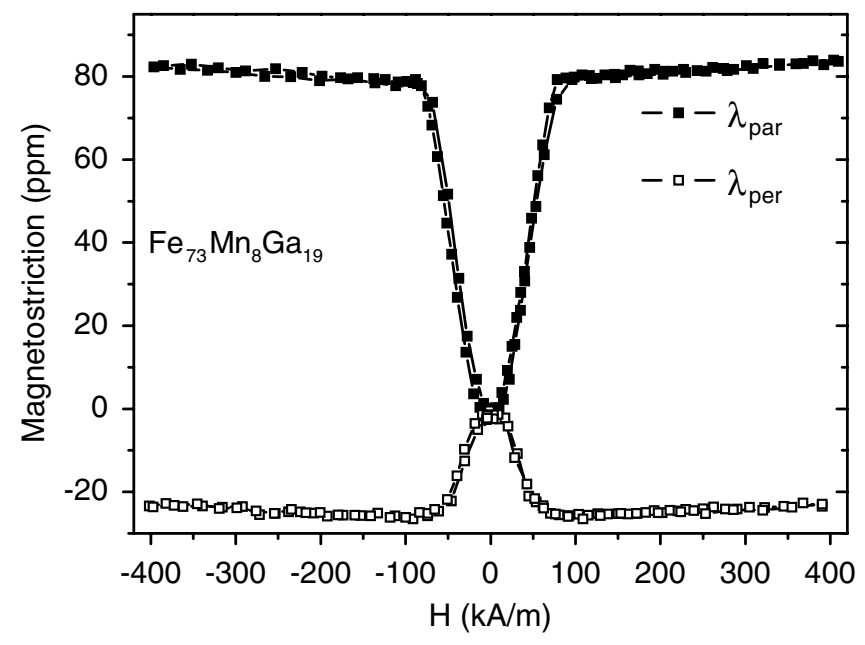

Figure 3. A typical magnetostriction measured parallel and perpendicular to the magnetic field for the composition of $\mathrm{Fe}_{73} \mathrm{Mn}_{8} \mathrm{Ga}_{19}$ at room temperature.

The Curie temperatures were determined from ac magnetic susceptibility measurements at high temperature. It is found that the Curie temperatures decrease with the substitution of $\mathrm{Mn}$ for $\mathrm{Fe}$ as expected, which is attributed to the smaller exchange interaction of $\mathrm{Mn}-\mathrm{Fe}$ than that of $\mathrm{Fe}-\mathrm{Fe}$ [15]. The general trend is depicted in figure $2(b)$.

Figure 3 shows a typical magnetostriction graph for composition $\mathrm{Fe}_{73} \mathrm{Mn}_{8} \mathrm{Ga}_{19}$, measured at room temperature, parallel and perpendicular to the applied magnetic field, respectively. The magnetostriction strain is positive for the parallel orientation $\left(\lambda_{\text {par }}\right)$ and a negative value is obtained for the perpendicular orientation $\left(\lambda_{\text {per }}\right)$. The magnetostriction values measured in these two directions are not very exactly consistent with the general expression $\left(\lambda_{\text {par }}=-2 \lambda_{\text {per }}\right)$ for them, probably due to slightly preferential orientation of the grains in the samples. Additionally, there is negligible 


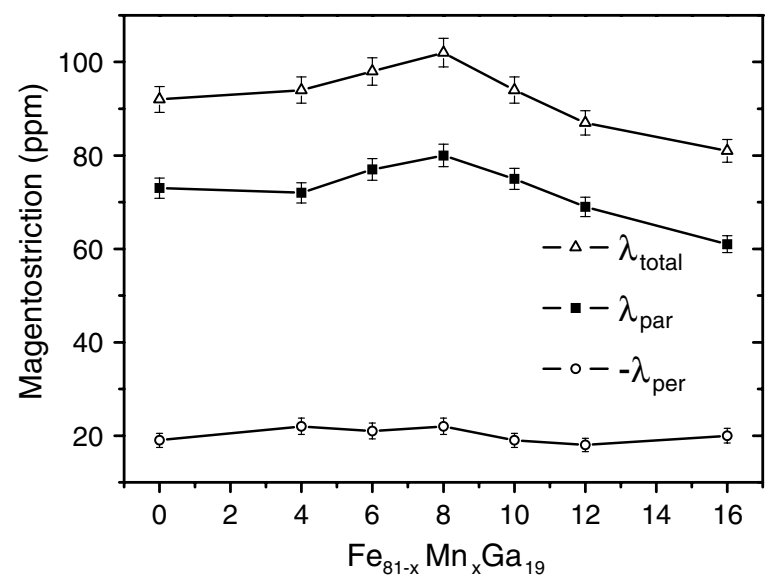

Figure 4. Mn content dependence of the parallel, perpendicular and total magnetostriction of $\mathrm{Fe}_{81-x} \mathrm{Mn}_{x} \mathrm{Ga}_{19}(x=0-16)$.

hysteresis in the magnetostriction against $H$ when $\mathrm{Mn}$ atoms are added, resulting in a reversible magnetostriction.

The equilibrium strain state of a crystal is determined by minimization of the sum of a crystal's anisotropic magnetic, magnetostrictive and elastic energies [16]. So in polycrystalline cubic materials, the general expression of $\lambda=3 / 2 \lambda_{\mathrm{s}}\left(\cos ^{2} \theta-1 / 3\right)$ is applicable. Here $\theta$ is the angle between the direction of the magnetic field and the measuring direction. $\lambda_{\mathrm{s}}$ is the saturation magnetostriction coefficient, which can be obtained by measuring $\lambda$ in a saturation field in parallel and perpendicular to the direction of measurement of magnetostriction. The total change obtainable in length caused by the magnetic field is then given by $\lambda_{\text {total }}=\lambda_{\text {par }}-\lambda_{\text {per }}=$ $3 / 2 \lambda_{\mathrm{s}}$, independent of the initial domain distribution. In our samples the highest obtainable value of the magnetostriction in a magnetically saturated state was obtained for the composition of $\mathrm{Fe}_{73} \mathrm{Mn}_{8} \mathrm{Ga}_{19}$, reaching a value of $102 \mathrm{ppm}$ at an applied field $H=80 \mathrm{kA} \mathrm{m}^{-1}$.

The variation of the parallel, perpendicular and total magnetostriction as a function of Mn concentration is shown in figure 4. A composition dependence of magnetostriction on the Mn concentration is observed along both directions. As can be seen, the largest magnetostriction improvement was obtained for $\mathrm{Fe}_{73} \mathrm{Mn}_{8} \mathrm{Ga}_{19}$, reaching a value of $11 \%$ larger than the value of $\mathrm{Fe}_{81} \mathrm{Ga}_{19}$. However, with a further increase in Mn concentration from $x=8$ to $x=16$, $\lambda_{\text {total }}$ decreases and finally it is about $12 \%$ less than the undoped sample. It can be considered that the magnetostriction performance in polycrystalline $\mathrm{Fe}-\mathrm{Ga}$ alloys can be improved by the substitutions of $\mathrm{Mn}$ for Fe. This improvement can be understood based on the dependence of the magnetostriction on the Akulov model [17].

The modelling of Akulov approximation has been used in the past to estimate the saturation magnetostriction $\lambda_{\mathrm{s}}$ of polycrystalline materials, which assumes no grain interaction and is equivalent to an average magnetostriction. As given in equation of $\lambda_{\mathrm{s}}=2 / 5 \lambda_{100}+3 / 5 \lambda_{111}, \lambda_{\mathrm{s}}$ is represented as a combination of the cubic magnetostriction constants. The recent study of Clark's results have indicated that the addition of $3 \mathrm{~d}$ transition elements of $\mathrm{Mn}$ reduce the saturation magnetostriction of $\lambda_{100}$, but the saturation magnetostriction

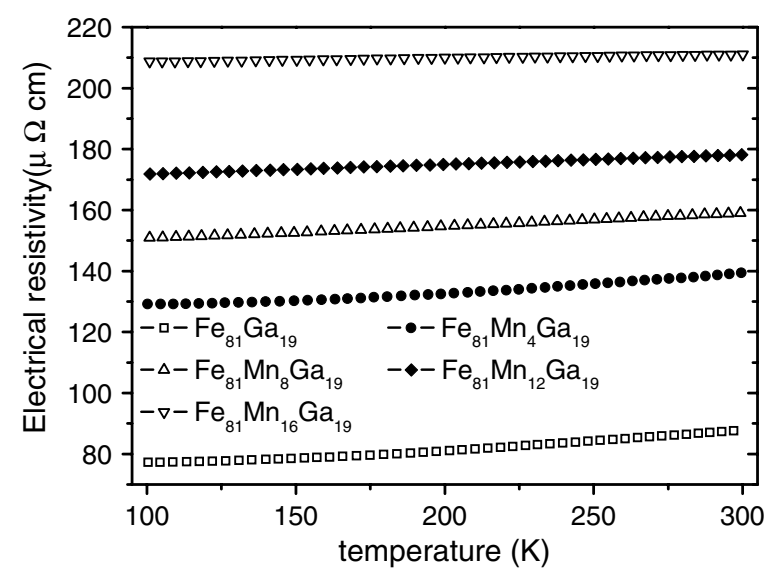

Figure 5. Temperature dependence of electrical resistivity of $\mathrm{Fe}_{81-x} \mathrm{Mn}_{x} \mathrm{Ga}_{19}(x=0-16)$.

$\lambda_{111}$ increases with Mn doping [18]. So the increase in the magnetostriction resulting from Mn doping in $x<8$ can be attributed to the increased magnetostriction in the $\lambda_{111}$ constant, which compensates for the decreased effect of $\lambda_{100}$. But with Mn doping more than $x=8$, the observation means that the decreasing of $\lambda_{100}$ becomes dominated and thus the saturation magnetostriction $\lambda_{\mathrm{s}}$ will decrease with the increase in Mn concentration. This further indicates that the Mn composition dependence of $\lambda_{100}$ and $\lambda_{111}$ are not the linearity, which results in a non-linear peak relation of $\lambda_{s}$ in our Mn-doped samples.

Temperature dependence of the electrical resistivity for Mn-doped Fe-Ga samples was measured in the temperature range from 100 to $300 \mathrm{~K}$, which is shown in figure 5. For all samples, the electrical resistivity exhibits a monotonic increase and shows a metallic behaviour. At room temperature, the electrical resistivity of arc-melted $\mathrm{Fe}_{81} \mathrm{Ga}_{19}$ is $88 \mu \Omega \mathrm{cm}$, which is basically in the same order of magnitude as Terfenol-D [7-9]. The resistivity rises to $159 \mu \Omega \mathrm{cm}$ for the $x=8$ and $211 \mu \Omega \mathrm{cm}$ for the $x=16$ sample, respectively, which is $81 \%$ and $140 \%$ larger than $\mathrm{Fe}_{81} \mathrm{Ga}_{19}$ alloy. The variation of resistivity at room temperature for $\mathrm{Fe}_{81-x} \mathrm{Mn}_{x} \mathrm{Ga}_{19}$ with $x$ is shown in figure $2(c)$.

Apparently, Mn addition is responsible for the increase in the electrical resistivity in our FeMnGa alloys. Intuitively, the random distributions of $\mathrm{Mn}$ atoms act as scattering centres for conduction electrons, and the electronic structure variation due to the addition of $\mathrm{Mn}$ with fewer conduction electrons than $\mathrm{Fe}$ is also considered to affect the electrical resistivity. Therefore, the electrical resistivity shows a positive composition dependence on Mn. The physical mechanism should be complex and detailed work for that is required in the future.

Increasing $\rho$ is good for reducing the eddy current in the magnetic materials. Furthermore, achieving this by doping $\mathrm{Mn}$, we also found the effective increase in the magnetostriction of $\mathrm{Fe}-\mathrm{Ga}$ alloys in quite a large range of $\mathrm{Mn}$ concentration. Our work suggests that $\mathrm{Mn}$ doped $\mathrm{Fe}-\mathrm{Ga}$ alloys may become an attractive candidate material for practical applications. 


\section{Conclusion}

In conclusion, the effects of $\mathrm{Mn}$ substitution for $\mathrm{Fe}$ on the magnetostriction and electrical resistivity of $\mathrm{Fe}_{81} \mathrm{Ga}_{19}$ have been investigated. An obvious increase in electrical resistivity, up to $140 \%$, is achieved for the $\mathrm{Fe}_{81-x} \mathrm{Mn}_{x} \mathrm{Ga}_{19}$ ( $x=0-16$ ) alloys without a great magnetostriction deterioration. We found that the substitution of Fe by $\mathrm{Mn}$ in the concentration of $x=0-8$ not only increases the electrical resistivity but also enhances the magnetostriction, about $11 \%$ larger than undoped alloys. It may make the $\mathrm{Fe}-\mathrm{Ga}$ alloys become very attractive candidate materials for magnetic actuator applications. The related physics is complex and require a further fundamental investigation.

\section{Acknowledgment}

This work was supported by the National Natural Science Foundation of China grant No 50531010.

\section{References}

[1] Kikuchi Y 1968 IEEE Trans. Magn. 4107

[2] Gibbs M R J 1992 Phys. Scr. T 45115

[3] Guruswany S, Srisukhumbowornchai N, Clark A E, Restorff J B and Wun-Fogle M 2000 Scr. Mater. 43239
[4] Srisukhumbowornchai N and Guruswany S $2001 \mathrm{~J}$. Appl. Phys. 905680

[5] Clark A E, Hathaway K B, Wun-Fogle M, Restorff J B, Lograsso T A and Keppens V M 2003 J. Appl. Phys. 938621

[6] Bormio-Nunes C, Tirelli M A, Turtelli R S, Grossinger R, Muller H and Wiesinger G 2005 J. Appl. Phys. 97033901

[7] Prajapati K, Jenner A G, Schulze M P and Greenough R D 1993 J. Appl. Phys. 736171

[8] Dhilsha K R and Rama-Rao K V S 1993 J. Appl. Phys. 731380

[9] Senthil-Kumar M, Reddy K V and Rama-Rao K V S 1995 J. Appl. Phys. 774589

[10] Landolt-Bornstein 1959 Zahlenwerte und Funktionen 6 Auflage, II band, 6 Teil (Berlin: Springer)

[11] Chelvane J A, Kasiviswanathan S and Markandeyulu G 2004 J. Magn. Magn. Mater. 277175

[12] Ikeda O, Kainuma R, Ohnuma I, Fukamichi K and Ishida K 2002 J. Alloys Compounds 347198

[13] Koster W and Godeke T 1977 Z. Metallk. 68661

[14] Kawamiya N, Adachi K and Nakamura Y 1972 J. Phys. Soc. Japan 331318

[15] Jaccarino V, Walker L R and Wertheim G K 1965 Phys. Rev. Lett. 13752

[16] Lee E W 1955 Rep. Prog. Phys. 18184

[17] Akulov N S 1930 Z. Phys. 66533

[18] Clark A E, Restorff J B, Wun-Fogle M, Hathaway K B, Lpgrasso T A, Huang M and Summers E 2007 J. Appl. Phys. 101 09C507 